\title{
Plasma Oscillations in Nanotransistors: Application to THz Radiations Detection and Generation
}

\author{
H. Marinchio*, G. Sabatini, C. Palermo, P. Nouvel, J. Torres, L. Varani \\ Institut d'Electronique du Sud, UMR 5214 CNRS, Université Montpellier 2, 34095 Montpellier, France
}

P. Shiktorov, E. Starikov and V. Gružinskis

Semiconductor Physics Institute, A. Goštauto 11, 2600 Vilnius, Lithuania

\begin{abstract}
By means of a numerical hydrodynamic model, we consider the mechanism of collective plasma oscillations in a field-effect transistor channel under different excitations and biasing conditions. First, we consider the case of a device externally-excited by a harmonic optical beating or an electronic excitation under constant current condition at the drain. Both situations exhibit sharp resonances related to the first odd plasma modes illustrating the possibility of using the HEMT as a terahertz photomixer or detector. Then, we demonstrate that the frequencies, amplitudes and quality factors of the resonances can be strongly modified by varying the drain biasing condition from current- to voltage-driven operation.
\end{abstract}

PACS: $72.15 . \mathrm{Nj}, 72.20 . \mathrm{Ht}, 72.30 .+\mathrm{q}$

\section{Introduction}

In the early 1990s, the theoretical work of Dyakonov and Shur have shown the possibility to sustain collective oscillations in a resonant cavity constituted by a field-effect transistor (FET) channel [1]. With modern short gate-length transistors, the frequency of these plasma wave oscillations, tunable by varying the gate length, can fall in the terahertz $(\mathrm{THz})$ range.

Since this pioneering work, two-dimensional plasma waves in quasi-ballistic submicron and nanometric transistors are considered a very promising physical phenomenon for the detection and generation of $\mathrm{THz}$ radiations $[2,3]$. Broadband THz emission due to the instability of the 2D electron gas of the channel of high-electron mobility transistors (HEMT) has been reported at cryogenic temperature for InGaAs channels [2] and at room-temperature with GaN devices [4].

Other theoretical and experimental works demonstrated that the plasma oscillation modes can be externally excited at room temperature by a $\mathrm{THz}$ radiation $[3,5]$ or by a THz optical beating $[6-8]$. In the former case, the transistor can act as a THz radiation detector while in the latter case, it can be used as a $\mathrm{THz}$ photomixer.

As it was recently demonstrated, the efficiency of such devices were significantly improved by driving the transistor in far-from-equilibrium regime $[8,9]$. That is why to perform a complete and accurate theoretical study of the plasma mechanism in FETs or HEMTs, a sufficiently complex numerical model is needed to overcome the limi-

\footnotetext{
* corresponding author; e-mail:

hugues.marinchio@ies. univ-montp2.fr
}

tations of usual models which, by assuming uniform profiles of carrier density, drift velocity, electric potential and the absence of hot-carrier effects $[1,10,11]$, are unable to describe quantitatively the plasma effects under strong drain-source biases.

Therefore, here we present a theoretical approach to investigate the phenomena of plasma wave excitation based on a pseudo-two-dimensional hydrodynamic (HD) scheme. As we shall demonstrate, such a model allows us to simulate nanometric FETs/HEMTs from ohmic to saturation conditions by taking into account nonequilibrium effects, presence of access regions, etc. [12]. The two different means - electrical and optical - to excite the plasma modes as well as the influence of the biasing conditions on the plasma effects will be discussed.

\section{Theoretical model}

The model consists of: (i) HD equations in the transport direction, here taken as the $x$-axis, relative to the electron density $n(x, t)$, the mean velocity $v(x, t)$ and the mean energy $\epsilon(x, t)$ according to [13] coupled with (ii) pseudo-2D approximation of the Poisson equation described in [14]:

$$
\left\{\begin{array}{l}
\frac{\partial n}{\partial t}=\frac{\partial(n v)}{\partial x}+G+\delta G \\
\frac{\partial v}{\partial t}=-v \frac{\partial v}{\partial x}-\frac{e E}{m^{*}}-\frac{1}{n} \frac{\partial\left(\overline{\delta v^{2}} n\right)}{\partial x}-v \nu_{v} \\
\frac{\partial \epsilon}{\partial t}=-v \frac{\partial \epsilon}{\partial x}-e E v-\frac{1}{n} \frac{\partial(\overline{\delta v \delta \epsilon})}{\partial x}-\left(\epsilon-\epsilon_{0}\right) \nu_{\epsilon} \\
\varepsilon_{c} \frac{\partial^{2} V}{\partial x^{2}}+\varepsilon_{s} \frac{V_{g}+\delta V_{g}-V}{d \delta}=e\left(n-N_{\mathrm{D}}^{\prime}\right)
\end{array}\right.
$$

Here $e$ is the elementary charge, $E$ is the electric field component along the channel, $\epsilon_{0}$ is the equilibrium mean energy, $\varepsilon_{s}$ and $\varepsilon_{c}$ are the dielectric constants of, respectively, the Schottky layer (for the case of a HEMT or 
a MESFET) or the oxide layer (for the case of a MOS$\mathrm{FET}), d$ is the gate-to-channel distance, $\delta$ is the channel thickness, $N_{\mathrm{D}}^{\prime}$ is the effective donor concentration in the channel [9]. The velocity and energy relaxation rates $\nu_{v}$, $\nu_{\epsilon}$, the electron effective mass $m^{*}$, the velocity variance $\overline{\delta v^{2}}$ and the energy-velocity covariance $\overline{\delta v \delta \epsilon}$ depend on the local mean energy and they were calculated by a Monte Carlo simulation of InGaAs bulk material. The photogenerated holes are supposed to be practically removed immediately from the channel to the gate, that is why their contribution is omitted in our model.

The model includes two external source terms:

(i) a beating optical excitation through the term $G+$ $\delta G=G_{0}[1+\cos (2 \pi f t)][12]$, and,

(ii) an electric excitation on the gate, which, for example, can describe the coupling between a $\mathrm{THz}$ radiation and an antenna connected to the gate [11], $\delta V_{g}=$ $\Delta V_{g} \cos (2 \pi f t)$. We will restrict our study to the cases of a harmonic excitation at $f$ frequency.

To simulate the constant current operation, an additional equation accounting for a large resistance $r$ connected in series with the HEMT source-drain channel has been used

$$
V_{d s}(t)=V_{T}-J_{d}(t) r,
$$

where $r$ is the resistance divided by the surface section of the channel, $V_{T}$ is the constant voltage applied to the whole source-drain- $r$ circuit, $J_{d}(t)$ and $V_{d s}(t)$ are the instantaneous total current and potential at the drain terminal, calculated by the numerical procedure. Such a model of an external circuit allows us to simulate both operations, namely, voltage-driven $(r \rightarrow 0)$ and current-driven $(r \rightarrow \infty)$ regimes.

We simulate an $\operatorname{In}_{0.53} \mathrm{Ga}_{0.47} \mathrm{As} / \mathrm{InAlAs}$ HEMT $\left(\varepsilon_{c}=\right.$ $13.4 \varepsilon_{0}, \varepsilon_{s}=11.8 \varepsilon_{0}, d=22 \mathrm{~nm}, \delta=10 \mathrm{~nm}$ ) composed of a $L_{g}=100 \mathrm{~nm}$ long gated part $\left(N_{\mathrm{D}}^{\prime}=1 \times 10^{18} \mathrm{~cm}^{-3}\right)$ surrounded by two $50 \mathrm{~nm}$ long ungated sections $\left(N_{\mathrm{D}}^{\prime}=\right.$ $\left.5 \times 10^{18} \mathrm{~cm}^{-3}\right)$. The threshold voltage $V_{\mathrm{th}}$ calculated by the Poisson equation in (1) gives a value $V_{\mathrm{th}}=-\frac{e N_{\mathrm{D}}^{\prime} d \delta}{\varepsilon_{s}}=$ $-300 \mathrm{mV}$ and, as a consequence, the swing voltage is $V_{0}=V_{g}-V_{\mathrm{th}}=0-(-300)=300 \mathrm{mV}$.

\section{Optical and electric excitations}

In this section, we consider the response of the previously described transistor to an optical beating and an electronic excitation.

We shall represent the total $V_{d s}(t)$ response to an external harmonic excitation at frequency $f$ as a sum of

$$
V_{d s}(t)=V_{d s}^{0}+\Delta V_{d s}(f)+\delta V(f) \cos (2 \pi f t+\phi(f)) .
$$

Here, $V_{d s}^{0}$ is the drain voltage without excitation, $\Delta V_{d s}(f)$ is the drain voltage dc component due to the excitation with the exciting frequency $f$, and the last term presents the ac component, that is the harmonic response. Let us note that in Eq. (3) the higher-order harmonics responses are omitted.

In the framework of such a representation it is convenient to characterize in the following the dc response as:
$\overline{\Delta V(f)}=\Delta V_{d s}(f)-\Delta V_{d s}(0)$. The calculated $\delta V(f)$ and $\overline{\Delta V(f)}$ are reported as functions of the beating frequency and for different values of $V_{\mathrm{DS}}=V_{d s}^{0}+\Delta V_{d s}(0)$ which is independent of the frequency $f . \overline{\Delta V(f)}$ is the interesting quantity to characterize the detection of a $\mathrm{THz}$ radiation or optical beating while $\Delta V_{d s}(f)$ describes the potential generation of $\mathrm{THz}$ electronic oscillations by the device.

Firstly, we illustrate the response of the channel to an external beating interband photoexcitation: $\Delta V_{g}=0$ and $G_{0}$ is taken equal to $1 \times 10^{27} \mathrm{~cm}^{-3} / \mathrm{s}$ which corresponds to typical conditions of photomixing experiments [9]. The harmonic and average photoresponses are reported in Fig. 1a and b, respectively. For both quantities, two resonances corresponding to the two first plasma modes are observed at $\mathrm{THz}$ frequencies.
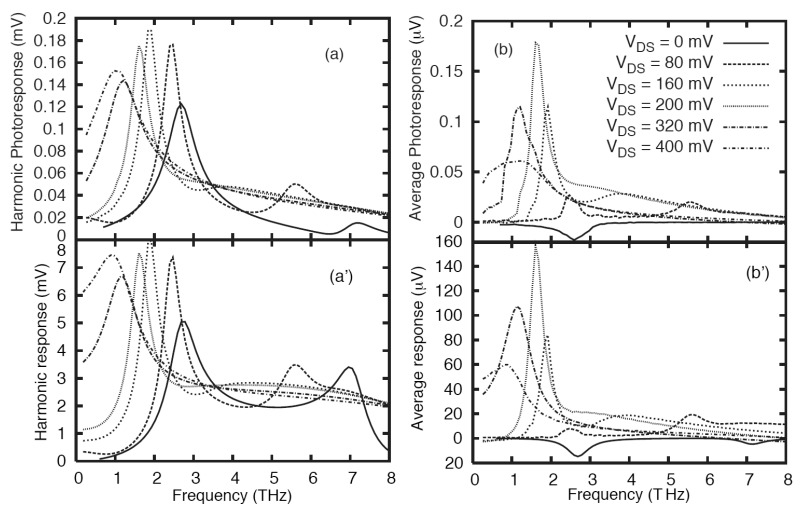

Fig. 1. Harmonic (a) and average (b) responses to a beating illumination or to an electronic excitation $\left(\mathrm{a}^{\prime}\right.$ and $\mathrm{b}^{\prime}$ ) as functions of the excitation frequency $f$ for different $V_{\mathrm{DS}}$.

Secondly, the optical excitation is substituted by an electronical harmonic oscillation of the gate-source voltage: $G_{0}=0$ while $\Delta V_{g}=1 \mathrm{mV}$. Figures $1 \mathrm{a}^{\prime}$ and $\mathrm{b}^{\prime}$ show the frequency-dependent harmonic and average responses of the transistor to the harmonic oscillating gate voltage for different $V_{\mathrm{DS}}$. The behavior is very similar to that observed in the case of optically excited channels: the same resonance frequencies are observed at the different drain voltages and the variation of resonance qualities with the drain bias is identical. This shows that these main characteristics of the excited plasma modes depends only on static parameters (gate length, gate and drain biases) and not on the nature of the external excitation. In practice, this implies that the process of optimization and tunability of a photomixing emitter or of a radiation detector will be quite similar. Moreover, this opens the possibility of an heterodyne detection based on the simultaneous electronic and optical excitations in a short FET [15].

\section{From ohmic to saturation regime}

We demonstrate here the influence of the drain bias on the amplitudes and frequencies of the plasma resonances. 
We only treat the case of the optical excitation since as follows from above, the similar results hold for electric excitation of plasma waves.

As seen in Fig. 1, at low voltage, the resonance frequency matches with the fundamental plasma frequency $f_{\mathrm{p}}=s / 4 L_{g} \approx 2.7 \mathrm{THz}$ (with $s=\sqrt{e V_{0} / m^{*}\left(\epsilon_{0}\right)} \approx$ $11 \times 10^{5} \mathrm{~m} / \mathrm{s}$ the plasma wave velocity), calculated with the assumption of a drift velocity much lower than the plasma velocity [1]. At increasing $V_{\mathrm{DS}}$, the resonance frequency of the peaks decreases significantly. This can be explained by the global growth of the mean velocity in the channel leading to an increase of the plasma transit time along the channel. Moreover, we observe that the amplitude and the quality factor of the resonant response can be maximized by playing on the drain voltage. As discussed in [12], this effect is mainly due to the optimization of the wave increment of the forced oscillator constituted by the photoexcited channel.

Let us point out that, in such short devices, transport is strongly non-local, which means that the drift velocity reaches very high values - over the saturation velocity - and becomes comparable to the plasma velocity for moderate or high drain voltage. Therefore, the increase of this bias has a great influence on the phase velocity and, consequently, on the fundamental frequency and the reflection coefficient of the wave at the drain junction $[1,12]$.

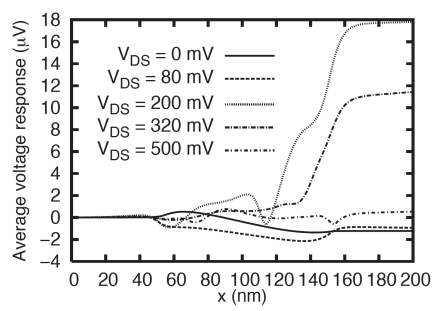

Fig. 2. Average response at the fundamental plasma resonance frequency as a function of the position $x$ along the device for different $V_{\mathrm{DS}} . G_{0}=10^{28} \mathrm{~cm}^{-3} / \mathrm{s}$ and $\Delta V_{g}=0 \mathrm{mV}$.

The fact that the velocity and velocity-gradient can become locally very strong near the drain contact has also a dramatic influence on the process of rectification of the plasma oscillation. Indeed, Fig. 2 represents the local average electric potential response, defined as the difference between the mean value of the potential under beating photoexcitation and its (static) value in absence of excitation $\Delta V(x)=\overline{V(x, t)}-V(x)_{\delta G=0}$, along the channel. For low drain-source bias, the rectification is distributed all along the channel leading to an additional voltage $\Delta V(x)$ with a sinusoidal shape demonstrating that the appearance of a (negative) average drain response $(\overline{\Delta V(f)})$ is due to the intrinsic non-linearity of the plasma mechanism as described in $[5,11]$. For moderate or high voltages, the growth of the mean voltage is localized at the vicinity of the drain contact where a depleted zone, characterized by a strong local electric field increase appears.
This indicates that, far-from-equilibrium, the rectification is controlled by the non-uniformity of the device itself. This process appears to be much more efficient than the one occurring in the ohmic regime. It explains why, at room-temperature experiments of photoexcitation of HEMTs, the plasma modes are more easily showed up in far-from-equilibrium conditions $[8,9]$.

\section{From current- to voltage-driven regime}

In this section, we consider a transistor with a resistive load of finite-value $r$ which is connected in series with the source-to-drain voltage source. By varying the value of the resistance $r$ from 0 to $\infty$ in Eq. (2), one can obtain a continuous transition from the voltage-driven to current-driven operations of the source-drain channel. This also allows us to consider at once the voltage and current responses due to optical beating excitation at frequency $f$.

Figure 3 shows the harmonic photoresponses for different values of $r / r_{d}$, where $r_{d}=\mathrm{d}\left\langle J_{d}\right\rangle / \mathrm{d}\left\langle V_{d s}\right\rangle$ is the dynamic resistance of the HEMT at $V_{0}=300 \mathrm{mV}$, from drain current-driven condition $\left(r \gg r_{d}\right)$ to voltage-driven condition $(r=0)$. By playing on the total voltage $V_{T}$ defined in Eq. (2), $V_{\mathrm{DS}}$ is fixed at $40 \mathrm{mV}$. Evidently, by decreasing the resistance $r$ and keeping $V_{\mathrm{DS}}$ constant, the current oscillation grows while the voltage one is reduced. More interestingly, the two resonance frequencies are changed by this modification of operation regime.

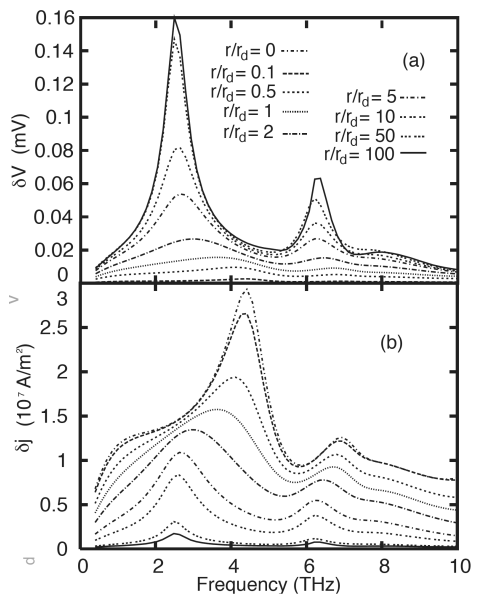

Fig. 3. Amplitude of the drain voltage (a) or current density (b) oscillation induced by the optical beating as a function of the beating frequency $f$ for different values of the resistive load. For $V_{\mathrm{DS}}=40 \mathrm{mV}, r_{d}=$ $10^{-12} \Omega \mathrm{m}^{-2}$.

Indeed, the asymmetric biasing conditions (drain current and source voltage fixed) imposed that the wavelength of the modes are odd submultiples of $4 L_{g}$, while the symmetric conditions only allow modes wavelength submultiples of $2 L_{g}$. That is why, at decreased $r$, the first mode frequency increases from $2.7 \mathrm{THz} \approx s / 4 L_{g}$ to $4.5 \mathrm{THz} \approx s / 2 L_{g}$ and the second resonance frequency from $6.3 \mathrm{THz} \approx 3 \times s /\left(4 L_{g}\right)$ to $7 \mathrm{THz} \approx 2 \times s / 2 L_{g}$. 
The small discrepancies between the numerical calculation and the analytical prediction from [1] can be explained by the dispersivity of the channel taken into account in our model [14].

Thus, the device appears to be tunable by playing on the drain load. Nevertheless, let us point out that the quality factor is optimal (i.e. the resonances are sharpest) in current-driven regime and decreases with the resistance. This is due to the amplification of the plasma wave at the reflection on a high impedance as described in [1].

\section{Conclusion}

We studied the two different ways of exciting plasma waves of a FET channel. This theoretical investigation is carried out by means of a original hydrodynamic pseudo-2D numerical model. We demonstrate that the first plasma resonances can be efficiently excited by optical or electronic excitations illustrating the possibility of using short gate-length HEMTs as $\mathrm{THz}$ photomixers or detectors operating at room temperature. The behavior of such devices is predicted to be highly dependent on the biasing conditions applied to the channel. Indeed, we demonstrated that the oscillation at the drain induced by the excitation of a plasma wave can be controlled and tuned by playing on the drain-source bias or the load connected to the transistor.

\section{Acknowledgments}

This work was partially supported by grant No. MIP$-87 / 2010$ of Lithuanian Science Council.

\section{References}

[1] M. Dyakonov, M. Shur, Phys. Rev. Lett. 71, 2465 (1993).

[2] W. Knap, F. Teppe, Y. Meziani, N. Dyakonova, J. Lusakowski, F. Boeuf, T. Skotnicki, D. Maude, S. Rumyantsev, M.S. Shur, Appl. Phys. Lett. 85, 675 (2004).
[3] J. Lusakowski, W. Knap, N. Dyakonova, L. Varani, J. Mateos, T. Gonzalez, Y. Roelens, S. Bollaert, A. Cappy, K. Karpierz, J. Appl. Phys. 97, 064307 (2005).

[4] A.E. Fatimy, R. Tauk, S. Boubanga, F. Teppe, N. Dyakonova, W. Knap, J. Lyonnet, Y.M. Meziani, T. Otsuji, M. Poisson, E. Morvan, S. Bollaert, A. Shchepetov, Y. Roelens, C. Gaquiere, D. Theron, A. Cappy, Phys. Status Solidi C 5, 244 (2008).

[5] M. Dyakonov, M.S. Shur, IEEE Trans. Electron Devices 43, 380 (1996).

[6] V. Ryzhii, I. Khmyrova, M. Shur, J. Appl. Phys. 91, 1875 (2002)

[7] T. Otsuji, M. Hanabe, O. Ogawara, Appl. Phys. Lett. 85, 2119 (2004).

[8] P. Nouvel, H. Marinchio, J. Torres, C. Palermo, L. Chusseau, D. Gasquet, L. Varani, P. Shiktorov, E. Starikov, V. Gružinskis, J. Appl. Phys. 106, 013717 (2009).

[9] J. Torres, H. Marinchio, P. Nouvel, G. Sabatini, C. Palermo, L. Varani, L. Chusseau, P. Shiktorov, E. Starikov, V. Gruzhinskis, IEEE J. Sel. Top. Quantum Electron. 14, 491 (2008).

[10] A.P. Dmitriev, A.S. Furman, V.Y. Kachorovskii, Phys. Rev. B 54, 14020 (1996).

[11] D. Veksler, F. Teppe, A.P. Dmitriev, V.Y. Kachorovskii, W. Knap, M.S. Shur, Phys. Rev. B $\mathbf{7 3}$ 125328 (2006).

[12] H. Marinchio, G. Sabatini, C. Palermo, J. Pousset, J. Torres, L. Chusseau, L. Varani, E. Starikov, P. Shiktorov, V. Gružinskis, Appl. Phys. Lett. 94, 192109 (2009).

[13] V. Gruzinskis, E. Starikov, P. Shiktorov, L. Reggiani, L. Varani, J. Appl. Phys. 76, 5260 (1994).

[14] P. Shiktorov, E. Starikov, V. Gružinskis, L. Varani, G. Sabatini, H. Marinchio, L. Reggiani, J. Stat. Mech. 01, 01047 (2009).

[15] H. Marinchio, L. Chusseau, J. Torres, P. Nouvel, L. Varani, G. Sabatini, C. Palermo, P. Shiktorov, E. Starikov, V. Gružinskis, Appl. Phys. Lett. 94, 013502 (2010). 